\title{
Kerr nonlinear coupler and entanglement induced by broadband laser light
}

\author{
Doan Quoc Khoa ${ }^{* 1}$, Cao Long Van ${ }^{2}$, Chu Van Lanh ${ }^{3}$, Nguyen Thanh Vinh ${ }^{3}$, Tran Thi Hai ${ }^{4}$, Ha Kim Quy ${ }^{4}$, and Nguyen \\ Thi Hong Sang 5 \\ ${ }^{1}$ Quang Tri Teacher Training College, Km3, Highway No 9, Dong Ha, Quang Tri, Viet Nam \\ ${ }^{2}$ Institute of Physics, University of Zielona Góra, Prof. A. Szafrana 4a, 65-516 Zielona Góra, Poland \\ ${ }^{3}$ Vinh University, 182 Le Duan, Vinh, Viet Nam \\ ${ }^{4}$ Hong Duc University, 565 Quang Trung, Thanh Hoa, Viet Nam \\ ${ }^{5}$ Tran Quoc Toan High School, Dong Thap, Viet Nam
}

Received August 27, 2016; accepted September 28, 2016; published September 30, 2016

\begin{abstract}
We consider the model of a Kerr-like nonlinear coupler linearly excited by an external classical field, involving a coherent part and white noise, solving a set of coupled stochastic integro-differential equations, and deriving exactly analytical formulae for the probability amplitudes of n-photon Fock states. As a consequence, the nonlinear coupler in question behaves as a two-qubit system. The effects of dissipation on entanglement of formation parameterised by concurrence are also analyzed and the results compared with those obtained previously in the literature. Interestingly, initially for a period of time the entanglement is enlarged when the chaotic parameter increases.
\end{abstract}

One of the central problems in quantum information theory is quantum state engineering which leads to finitedimensional states generation. Such states can exhibit several interesting properties as they are able to produce various kinds of quantum correlations. The most important type of these for quantum computing is quantum entanglement. It is one of the most basic concepts of the theory of quantum information and allows us to study many features of nonlocal properties of quantum systems [1]. Different aspects of entanglement and its generation have been considered in numerous works.

Quantum optical systems based on Kerr nonlinearities are those which allow for creation of entangled states. Such a system can be found in various physical situations and referred to as Kerr-like couplers. It has been shown that the quantum evolution of the system can be closed in a two-qubit Hilbert space and can lead to Bell states generation [2-3]. It is shown that the coupler evolution governed by the same effective Hamiltonians as usual optical Kerr couplers (discussed for instance in [4]) can be closed within a finite set of $n$-photon states. After "truncation" of the wave function describing the system, the coupler can be treated as two-qubit and treated as a Bell-like state generator that can create maximally entangled states even if the system exhibits its energy dissipating nature [5]. The system behaves similar to nonlinear quantum scissors and maximally entangled Bell states can be generated with high efficiency [6].

*E-mail: khoa_dqspqt@yahoo.com
In previous papers, including these mentioned above, it was assumed that the laser light is monochromatic. However, a real laser is never perfectly monochromatic and it is interesting to include fluctuations of a laser beam. In the present paper we model laser beam fluctuations with a Gaussian process.

The exact analytical averaging of stochastic equations with a Gaussian process is a difficult task. Hence, it is worthwhile to start with an extreme case of white noise. Even in this case we can obtain several interesting results [7-9]. Here we extend the formalism given in [10] to a more realistic case, when the laser width is taken into account and discuss a simple quantum information application of Kerr nonlinearities, namely for generation of entangled optical qubits from classical light. We consider here a simple model including two quantum nonlinear oscillators located inside one cavity. These oscillators are linearly coupled to each other, while one of the oscillators is excited by an external coherent field, which is assumed to be decomposed into two parts: a coherent part and white noise. Then a set of stochastic differential equations of motion describing the system dynamics will be obtained and analytically solved. The plot of probabilities corresponding to the Bell-like states and concurrence for the excited nonlinear coupler will be calculated analytically and compared with the results obtained in [10].

In this paper we investigate the model of a Kerr-like nonlinear coupler comprising two nonlinear oscillators linearly coupled to each other. One of these nonlinear oscillators is coupled to an external classical field. Such a system is depicted by the Hamiltonian of the form [10]:

$\hat{H}=\frac{\chi_{a}}{2}\left(\hat{a}^{+}\right)^{2} \hat{a}^{2}+\frac{\chi_{b}}{2}\left(\hat{b}^{+}\right)^{2} \hat{b}^{2}+\varepsilon \hat{a}^{+} \hat{b}+\varepsilon^{*} \hat{a} \hat{b}^{+}+\alpha \hat{a}^{+}+\alpha^{*} \hat{a}$,

in which $\hat{a}^{+}(\hat{b})^{+}$and $\hat{a}(\hat{b})$ are the boson creation and annihilation operators, respectively, the parameters $\chi_{a}$ and $\chi_{b}$ respectively are the constants of nonlinearity of the oscillators $a$ and $b, \alpha$ is the strength of the external 
excitation of the oscillator $a$, while $\varepsilon$ is the strength of the oscillator-oscillator coupling.

We can write the wave function describing our model in the form:

$$
|\psi(t)\rangle=c_{00}(t)|0\rangle_{a}|0\rangle_{b}+c_{10}(t)|1\rangle_{a}|0\rangle_{b}+c_{01}(t)|0\rangle_{a}|1\rangle_{b}+c_{11}(t)|1\rangle_{a}|1\rangle_{b} .
$$

We suppose that the amplitude of an external classical field involving two parts: coherent part and white noise and we shall restrict ourselves to the case of real $\alpha_{0}=\varepsilon$. Moreover, we also suppose that for the time $t=0$ both oscillators are in the states of vacuum. Thus, we derive a system of equations for stochastic averages of variables and its solutions for the probability amplitudes $c_{i j}(i, j=0,1)$ in the form: (the volume of this communication is limited, so we shall concentrate here only on physical aspects of the problem, whereas the mathematical details of this procedure will be described elsewhere [11]):

$$
\begin{aligned}
c_{00}(t) & =\frac{e^{-\frac{i a_{0}}{4} t}}{2 \sqrt{5}}\left[(\sqrt{5}+1) \cos \frac{x_{2} t}{4}+(\sqrt{5}-1) \cos \frac{x_{1} t}{4}\right] \\
& +\frac{i a_{0} e^{-\frac{i a_{0}}{4} t}}{2 \sqrt{5}}\left[\frac{\sqrt{5}+1}{x_{2}} \sin \frac{x_{2} t}{4}+\frac{\sqrt{5}-1}{x_{1}} \sin \frac{x_{1} t}{4}\right], \\
c_{01}(t) & =\frac{e^{-\frac{i a_{0}}{4} t}}{\sqrt{5}}\left[\cos \frac{x_{1} t}{4}-\cos \frac{x_{2} t}{4}\right]+\frac{i a_{0} e^{-\frac{i a_{0}}{4} t}}{\sqrt{5}}\left[\frac{1}{x_{1}} \sin \frac{x_{1} t}{4}-\frac{1}{x_{2}} \sin \frac{x_{2} t}{4}\right], \\
c_{10}(t) & =-\frac{2 i \varepsilon e^{-\frac{i a_{0}}{4} t}}{\sqrt{5}}\left[\frac{\sqrt{5}+1}{x_{1}} \sin \frac{x_{1} t}{4}+\frac{\sqrt{5}-1}{x_{2}} \sin \frac{x_{2} t}{4}\right], \\
c_{11}(t) & =-\frac{4 i \varepsilon e^{-\frac{i a_{0}}{4} t}}{\sqrt{5}}\left[\frac{1}{x_{1}} \sin \frac{x_{1} t}{4}-\frac{1}{x_{2}} \sin \frac{x_{2} t}{4}\right],
\end{aligned}
$$

where $\alpha_{0}$ is the deterministic coherent part of the external classical field, $\varepsilon$ is the strength of the oscillator-oscillator coupling, $a_{0}$ is the chaotic parameter, $x_{1}=\sqrt{a_{0}^{2}+4 \Omega_{1}^{2}}$, $x_{2}=\sqrt{a_{0}^{2}+4 \Omega_{2}^{2}}, \Omega_{1}=\varepsilon \sqrt{2(3+\sqrt{5})}$ and $\Omega_{2}=\varepsilon \sqrt{2(3-\sqrt{5})}$.

We shall express the obtained wave function in the Bell basis

$$
|\psi\rangle=b_{1}\left|B_{1}\right\rangle+b_{2}\left|B_{2}\right\rangle+b_{3}\left|B_{3}\right\rangle+b_{4}\left|B_{4}\right\rangle,
$$

in which the states $\left|B_{i}\right\rangle, i=1,2,3,4$ are Bell-like states. These states can be expressed as functions of the $n$-photon states considered here:

$$
\left|B_{1}\right\rangle=\frac{|11\rangle+i|00\rangle}{\sqrt{2}},\left|B_{2}\right\rangle=\frac{|00\rangle+i|11\rangle}{\sqrt{2}},\left|B_{3}\right\rangle=\frac{|01\rangle-i|10\rangle}{\sqrt{2}},\left|B_{4}\right\rangle=\frac{|10\rangle-i|01\rangle}{\sqrt{2}} .
$$

in which the states $\left|B_{i}\right\rangle, i=1,2,3,4$ are Bell-like states. These states are maximally entangled states, and thus, for the cases if $\left|b_{i}\right|^{2}=1, i=1,2,3,4$ then our system also evolves into an maximally entangled state.
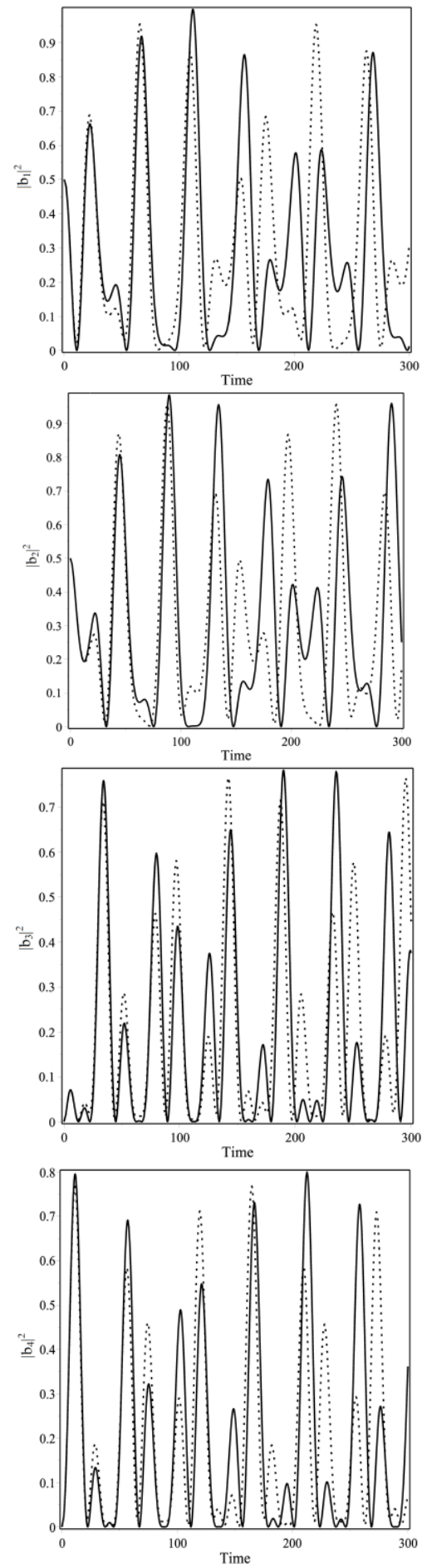

Fig. 1. Probabilities for finding the coupler in the Bell-like states. The coupling strengths $\varepsilon=\pi / 25$. Solid curve is for $a_{0}=0$, dotted curve is for $a_{0}=\pi / 30$.

Figure 1 describes the probabilities corresponding to the Bell-like states as a time function. When the chaotic component is absent $\left(a_{0}=0\right)$, we obtain the states $\left|B_{1}\right\rangle$ and $\left|B_{2}\right\rangle$ which are generated with high accuracy - our system 
becomes maximally entangled. This entanglement includes the states $|0\rangle_{a}|0\rangle_{b}$ and $|1\rangle_{a}|1\rangle_{b}$. However, if the chaotic component is present $\left(a_{0} \neq 0\right)$ then our system cannot be maximally entangled for the states $\left|B_{1}\right\rangle$ and $\left|B_{2}\right\rangle$. Moreover, when the chaotic component is absent, the values of probabilities for the states $\left|B_{3}\right\rangle$ and $\left|B_{4}\right\rangle$ can maximally achieve 0.8 , but if the chaotic component is present, they can only maximally reach 0.76 . As a consequence, for the initial vacuum states $|0\rangle_{a}|0\rangle_{b}$, the states $|1\rangle_{a}|0\rangle_{b}$ and $|0\rangle_{a}|1\rangle_{b}$ cannot be maximally entangled. However, the generation of $\left|B_{3}\right\rangle$ and $\left|B_{4}\right\rangle$ would be likely by supposing that the system is initially in the states $|1\rangle_{a}|0\rangle_{b}$ or $|0\rangle_{a}|1\rangle_{b}$. Thus, we can see that when the chaotic component is present, the population of maximally entangled states in our system reduces. Moreover, the maximum values of the probabilities decrease and shift towards the zero of time in comparison to the case when the chaotic component is absent.

To measure the entanglement degree of the system, we can use the concurrence that is one of the most commonly applied measures of entanglement. The concurrence for two-qubit states is defined as follows:

$$
C=\max \left\{0, l_{1}-l_{2}-l_{3}-l_{4}\right\},
$$

in which $l_{i}(i=1,2,3,4)$ are the square roots of the eigenvalues of the following matrix:

$$
\tilde{\eta}=\eta\left(\sigma_{y}^{a} \otimes \sigma_{y}^{b}\right) \eta^{*}\left(\sigma_{y}^{a} \otimes \sigma_{y}^{b}\right),
$$

where $\sigma_{y}^{(a, b)}$ are Pauli matrices acting in subspaces corresponding to the modes $\{a, b\}$, and the eigenvalues $l_{i}$ in (6) should be taken in a reducing order. Concurrence gets values from zero to one: for unentangled states it vanishes, while for maximally entangled states it is equal to one.

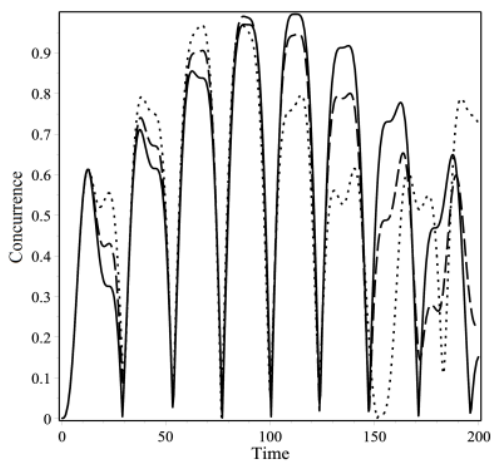

Fig. 2. Concurrence for the excited nonlinear coupler. The coupling strengths $\varepsilon=\pi / 25$. Solid curve is for $a_{0}=0$, dotted curve is for $a_{0}=\pi / 30$.

The plot of the concurrence evolution is shown in Fig. 2. Various curves in Fig. 2 correspond to concurrence evolutions with different values of the chaotic component $a_{0}$. When $a_{0}=0$, our result becomes exactly similar to that obtained in [10]. We see that if $a_{0}$ increases then the concurrence increases for $t<100$ and decreases for $t>100$. Thus, several maxima appear for $t<100$ and several maxima disappear for $t>100$ when $a_{0}$ increases.

As a consequence, the parameter $a_{0}$ related to the chaotic component is an important parameter which controls the appearing maxima of concurrence.

In conclusions, we have considered the model of a nonlinear coupler linearly excited by an external classical field which is assumed to be decomposed into two parts: a coherent part and white noise. We have shown that the evolution of the system is closed within a finite set of states and only $|i\rangle_{a}|j\rangle_{b}(i, j=0,1)$ states are populated. We have applied here the method used for the nonlinear quantum scissors [12] and have found some analytical formulae for the probability amplitudes corresponding to these states. We have shown that, starting from the vacuum state $|0\rangle_{a}|0\rangle_{b}$ of our system, its evolution leads to

Bell-like state generation. Moreover, we have calculated the concurrence and have shown that the concurrence exhibits some modulation effect as a result of the existence of various couplings in our system. We show that when the chaotic component is present, the populations of the maximally entangled states in our system decreases. Moreover, the maximum values of the probabilities decrease and shift towards the zero of time in comparison to the case when the chaotic component is absent. In addition, the concurrence of the system changes inversely, namely on the left of $t=100$ the concurrence increases but on the right of $t=100$ the concurrence decreases. As a consequence, the parameter $a_{0}$ related to the chaotic component is an important parameter which controls the maxima appearance of concurrence maxima. Moreover, in contradiction to our prediction, initially, for a period of time (equal 100) the entanglement is amplified when the chaotic component increases.

This research is funded by the Vietnam National Foundation for Science and Technology Development (NAFOSTED) under the grant number 103.03-2014.13.

\section{References}

[1] A. Einstein, B. Podolski, N. Rosen, Phys. Rev. 47, 777 (1935).

[2] A. Miranowicz, W. Leoński, J. Phys. B 39, 1683 (2006).

[3] T.D. Nguyen et al., Comput. Method. Sci. Tech. 19, 175 (2013).

[4] N. Korolkova, J. Peřina, Opt. Commun. 136, 135 (1997).

[5] A. Kowalewska-Kudłaszyk, W. Leoński, Phys. Rev. A 73, 042318 (2006).

[6] A. Kowalewska-Kudłaszyk et al., Phys. Scr. T 160, 014023 (2014).

[7] K. Doan Quoc, V. Cao Long, W. Leoński, Phys. Scr. T 147, 014008 (2012).

[8] K. Doan Quoc, V. Cao Long, W. Leoński, Phys. Scr. 86, 045301 (2012).

[9] K. Doan Quoc et al., Optica Applicata 46, 93 (2016).

[10] W. Leoński, A. Miranowicz, J. Opt. B 6, S37 (2004).

[11] K. Doan Quoc, V. Cao Long, to be published

[12] W. Leoński, Phys. Rev. A 55, 3874 (1997). 